\title{
Investigation of turbulence flow and sediment entrainment around a bridge pier
}

\author{
Elham Izadinia • Manouchehr Heidarpour • \\ Anton J. Schleiss
}

Published online: 28 November 2012

(c) Springer-Verlag Berlin Heidelberg 2012

\begin{abstract}
Local scour around the obstacles such as bridge pier is the result of complex interaction between turbulent flow and sediment particles at the mobile bed. The entrainment of sediment particles from the bed is stochastic in nature and it is strongly influenced by instantaneous shear stresses of the bursting process. The focus of this study is the investigation of turbulent flow and analysis of contribution of each bursting events to the Reynolds shear stress to find the dominant bursting events and also the flow structure around the circular bridge pier. The velocities around the bridge pier were measured in three dimensions using an Acoustic Doppler Velocimeter. These velocities were measured at different positions around the bridge pier and at different depths. Quadrant analysis is used to recognize the susceptible regions for sediment entrainment and deposition. According to quadrant analysis sedimentation is the dominant effect in the scour hole whereas at higher levels the erosion force becomes more important. In downstream of the pier, sediment particles are put in suspension and transported downstream due to sweep. Our results indicate that the secondary currents are more dominant in downstream of the pier, as compared to the upstream of the pier. Consequently the maximum velocity in downstream of the pier takes place in a
\end{abstract}

E. Izadinia $(\bowtie) \cdot$ M. Heidarpour

Department of Water Engineering, Agricultural College, Isfahan

University of Technology, Isfahan, Iran

e-mail: Elham.izadinia@epfl.ch; elham.izadinia@ag.iut.ac.ir

M. Heidarpour

e-mail: heidar@cc.iut.ac.ir

Present Address:

E. Izadinia - A. J. Schleiss

Laboratory of Hydraulic Constructions, Ecole Polytechnique

Fédérale de Lausanne, Lausanne, Switzerland

e-mail: anton.schleiss@epfl.ch location closer to the bed. In upstream of the pier the streamwise component of velocity becomes positive for which the universal log-law turns out to be valid.

Keywords Bridge pier · Bursting events - Quadrant analysis $\cdot$ Scour $\cdot$ Velocimetry

\begin{tabular}{|c|c|}
\hline \multicolumn{2}{|c|}{ Abbreviation } \\
\hline$\sigma_{\mathrm{g}}[-]$ & Geometric standard deviation of particles \\
\hline $\mathrm{d}_{50}[\mathrm{~L}]$ & Median diameter of sediment particles \\
\hline $\mathrm{D}[\mathrm{L}]$ & Pier diameter \\
\hline $\mathrm{Z}[\mathrm{L}]$ & $\begin{array}{l}\text { Vertical distance from the measuring point } \\
\text { to bed }\end{array}$ \\
\hline $\mathrm{h}[\mathrm{L}]$ & Total flow depth \\
\hline $\mathrm{u}\left[\mathrm{LT}^{-1}\right]$ & $\begin{array}{l}\text { Instantaneous velocities in the longitudinal } \\
\text { directions }\end{array}$ \\
\hline $\mathrm{v}\left[\mathrm{LT}^{-1}\right]$ & $\begin{array}{l}\text { Instantaneous velocities in span-wise } \\
\text { directions }\end{array}$ \\
\hline $\mathrm{w}\left[\mathrm{LT}^{-1}\right]$ & Instantaneous velocities in vertical directions \\
\hline$\overline{\mathrm{u}}\left[\mathrm{LT}^{-1}\right]$ & $\begin{array}{l}\text { Temporal mean velocities in the longitudinal } \\
\text { directions }\end{array}$ \\
\hline$\overline{\mathrm{w}}\left[\mathrm{LT}^{-1}\right]$ & $\begin{array}{l}\text { Temporal mean velocities in the vertical } \\
\text { directions }\end{array}$ \\
\hline $\mathrm{N}[-]$ & $\begin{array}{l}\text { The number of instantaneous velocity } \\
\text { samples }\end{array}$ \\
\hline $\mathrm{H}[-]$ & Hole size parameter \\
\hline $\mathrm{T}[\mathrm{T}]$ & Time interval \\
\hline$I_{H}^{i}(y, t)[-]$ & Detection function \\
\hline $\mathrm{W}[\mathrm{L}]$ & Channel width \\
\hline$u_{*}\left[\mathrm{LT}^{-1}\right]$ & The friction velocity \\
\hline $\mathrm{k}_{\mathrm{s}}[\mathrm{L}]$ & The equivalent standard roughness of the bed \\
\hline $\mathrm{B}_{\mathrm{R}}[-]$ & Constant of integration \\
\hline$\kappa[-]$ & Von Karman constant \\
\hline$\alpha[-]$ & Non-Gaussian parameter \\
\hline
\end{tabular}

\section{Abbreviation}

$\mathrm{d}_{50}[\mathrm{~L}]$

$\mathrm{D}[\mathrm{L}]$

Median diameter of sediment particles

$\mathrm{Z}[\mathrm{L}]$

Vertical distance from the measuring point

h [L]

Total flow depth

$\mathrm{u}\left[\mathrm{LT}^{-1}\right]$ directions

$\mathrm{v}\left[\mathrm{LT}^{-1}\right]$

Instantaneous velocities in span-wise

$\mathrm{w}\left[\mathrm{LT}^{-1}\right.$ ]

Instantaneous velocities in vertical directions

$\overline{\mathrm{u}}\left[\mathrm{LT}^{-1}\right]$ directions directions

The num
samples

$\mathrm{H}[-]$

Hole size parameter

$\mathrm{T}[\mathrm{T}]$

$I_{H}^{i}(y, t)[-]$

$\mathrm{W}[\mathrm{L}]$

Channel width

$u_{*}\left[\mathrm{LT}^{-1}\right]$

The friction velocity

$\mathrm{k}_{\mathrm{s}}[\mathrm{L}]$

$\mathrm{B}_{\mathrm{R}}[-]$

$\alpha[-]$ 


\section{Introduction}

Scouring due to the turbulent flow around bridge pier is one of the major causes of bridge failure. In addition, the eroded sediment is a significant component of many environmental degradation problems. The process of sediment transport, however, is very complicated due to the interaction of many parameters. Furthermore, scouring changes the morphology of the river and affects aquatic ecosystems and fish habitats. Fish communities in the rivers and streams are quite sensitive to the availability of stable pools and scour hole volume (Arlinghaus et al. 2002; Armstrong et al. 2003). Thus, investigating the hydrodynamics of the flow structure has attracted the interest of researchers in the field of environmental fluid mechanics and ecology.

In spite of the large number of experimental studies on local scouring that can be found in the literature, there is a lack of understanding of the coherent structures in turbulent flow around the bridge pier. Most of the investigations have focused on the countermeasure techniques to reduce the local scouring (e.g. Chiew 1992; Dey 1997; Kummar et al. 1999; Chiew and Lim 2000; Zarrati et al. 2004; Dey and Rajkumar 2007a; Moncada-M et al. 2009) or concentrated to predict the maximum scour depth (e.g. Melville and Coleman 2000; Barbhuiya and Dey 2004).

The stochastic nature of turbulent flow around bridge pier has been investigated only in a handful studies (e.g. Melville 1975; Dey et al. 1995; Istiarto 2001; Dey and Rajkumar 2007b); hence the precise effect of pier on the flow separation, reattachment, and the perturbed shear layer in the turbulent flow responsible for sediment transport is yet unclear.

Kline et al. (1967) have introduced the concept of bursting phenomenon as a mean to describe the transfer of momentum between the turbulent and laminar region near the boundary. Wallace et al. (1972) and Willmarth and Lu (1972) have applied a conditional sampling and averaging technique to quantify the contribution to the Reynolds shear stress during a cycle of events observed in the bed region of the turbulent boundary layer. Lu and Willmarth (1973) introduced the quadrant analysis for studying the structure of the bursting phenomenon. The quadrant analysis was employed to determine the frequency of occurrence of each individual event within a bursting process, i.e. outward interactions, ejections, inward interactions, and sweeps. Nakagawa and Nezu (1978) and Grass (1971, 1982) have shown that the sweep event above the channel bed is more responsible for transfer of momentum into the boundary layer than the ejection event. In addition, Nakagawa and Nezu (1978), Thorne et al. (1989), and Keshavarzy and Ball (1995) indicated that the sweep and ejection events occur more frequently than the outward interaction (quadrant $I$ ) and inward interaction (quadrant III) events. Williams (1990) and Thorne et al. (1989) showed that sediment entrainment occurs most frequently during sweep events and only occasionally during outward interaction events, whereas transport of suspended sediment depends primarily on the ejection event. Additionally, Keshavarzy and Ball (1995) have shown that the magnitude of the instantaneous shear stress in a sweep event is greater than outward and inward interaction events.

Although quadrant analysis is traditionally performed for conditional sampling of the Reynolds shear stress and turbulent heat flux, recent studies (e.g., Poggi et al. 2004; Ghisalberti and Nepf 2006; Zhu et al. 2007; Afzalimehr et al. 2011) extended the technique to include more general properties of turbulence in plant canopy flows, such as turbulent kinetic energy, vorticity, and dissipation rate. Jafari and Keshavarzi (2010) investigated turbulence flow and sediment entrainment over the ripples by using quadrant analysis. Esfahani and Keshavarzi (2011) analyzed the bursting events in meander channels with different curvatures to find the turbulent characteristics of flow and sediment motion in river bends. However, in spite of the importance of coherent structures of turbulence and in particular the bursting events around the bridge pier; their characteristics have not been investigated in detail. Because the quadrant analysis is a powerful technique to recognize the structure of the bursting phenomenon and consequently to find the susceptible regions for sediment entrainment and deposition, this technique is considered in present study to investigate the coherent structure of turbulent flow in scouring process around the bridge pier. In addition the effect of bridge pier on the mean flow, turbulence, and the occurrence probabilities of each bursting events are investigated. The main objective is to determine the contribution of each quadrant to the Reynolds shear stress and to compute the dominant event of turbulent structure around the bridge pier for better understanding of sediment transport in the scouring process.

\section{Materials and methods}

\subsection{Experimental setup}

The experiments were carried out in a smooth rectangular flume with $8 \mathrm{~m}$ length, $0.4 \mathrm{~m}$ width and $0.6 \mathrm{~m}$ height. The entrance of channel was filled with sand in order to generate fully developed flows. The working section, in which piers were located, was $1.6 \mathrm{~m}$ long with a recess on the bed $0.15 \mathrm{~m}$ deep and was situated $4 \mathrm{~m}$ downstream from the entrance of the flume. The recess was filled with uniform sediment with the mean particle sizes of $0.72 \mathrm{~mm}$ and geometric standard deviation of particles was $\sigma_{g}=1.12$. Uniform sand, having the same size as that used for the 
scouring test, was glued over the false floor (Fig. 1a). Based on Raudkivi and Ettema (1983), for non-rippleforming sediment $\left(d_{50} \geq 0.7 \mathrm{~mm}\right)$, experiments can be successfully run with a flow condition without disturbing the upstream bed by the approach flow. According to Chiew and Melville (1987) if geometric standard deviation of particles are lower than 1.3, the effect of non-uniformity of sediment on the depth of scour hole becomes negligible. The water depth in the flume is adjusted by the sluice gate in downstream of the flume.

A circular pier with diameter of $40 \mathrm{~mm}$ was used. According to Chiew and Melville (1987) the pier diameter should not be larger than $10 \%$ of channel width. Pier diameters were selected so that the effect of flume sidewalls on the depth of scour hole becomes negligible. Because $D / d_{50}>50\left(D / d_{50}=55.5\right.$ where $D$ is the pier diameter) the effect of sediment size on the depth of scour hole becomes negligible (Chiew and Melville 1987).

Since the maximum depth of scour in clear water condition occurs at the threshold of bed material motion, all tests were conducted at this condition (Raudkivi 1990). The threshold of bed material motion was found by performing a test before installing the pier. Threshold of material motion was defined as a condition such that while finer bed materials move, the overall average elevation of the bed is not lowered more than 2-3 $\mathrm{mm}$ during the period of the experiment. Three velocity profiles were measured by Acoustic Doppler Velocimeter (ADV) in test section along the centerline of the channel to obtain the flow intensity. These tests showed that with a flow depth of $0.16 \mathrm{~m}$ and a flow rate of $0.0192 \mathrm{~m}^{3} / \mathrm{s}$, the bed material would be at incipient motion condition. The ratio of the shear velocity to the critical shear velocity in our experiments was found to be approximately 0.9 .

Definition of equilibrium condition is complicated. Several researchers have studied time-dependent scour depth for clear water condition. They have introduced different criteria for equilibrium time. Kumar et al. (1999) stopped their experiments when the scour depth did not change by more than $1 \mathrm{~mm}$ over a period of $3 \mathrm{~h}$. Mia and Nago (2003) stopped their experiments when there was less than $1 \mathrm{~mm}$ scour by $1 \mathrm{~h}$. Melville and Chiew (1999) defined the equilibrium time when the scour depth did not change by more than $5 \%$ of the pier diameter over a period of $24 \mathrm{~h}$. In this study the equilibrium condition is obtained after $50 \mathrm{~h}$ according to Melville and Chiew's criteria.

A down-looking Acoustic Doppler Velocimeter (ADV), developed by Nortek, was used to measure instantaneous three dimensional velocity components. The ADV was installed on tracks above the flume; it was easily movable in vertical and horizontal directions. Errors in the prediction of mean velocities were less than $1 \%$. Velocities were recorded for each point with a sampling frequency of $200 \mathrm{~Hz}$ and a sampling duration of $120 \mathrm{~s}$. WinADV was used to filter and process the velocity and turbulence data. Data with an average correlation coefficient of less than $70 \%$ and signal to noise ratio (SNR) of less than $5 \mathrm{~dB}$ was filtered out.

Velocity profiles were measured approximately in 60 different longitudinal and transversal positions around the bridge pier. In each vertical profile at least 20 points were measured within the flow depth from the bed. Hence, the velocities at 1200 points within the flow around the bridge pier were analyzed. The lowest point for velocity measurements in each profile is being $3 \mathrm{~mm}$ above the bed. Also, the ADV used in this experimental study was not able to measure the velocity at a depth of $z / h>0.75$ (where $z$ is the vertical distance from the measuring point to bed; and $h$ is the total flow depth).

The ADV readings were taken when the scour hole was at equilibrium condition. To stabilize a scour hole in equilibrium condition, the cement was sieved uniformly over the scoured bed (Fig. 1b). After the bed became indestructible, ADV measurements were performed (Fig. 1c).

\subsection{Quadrant analysis}

The bursting process consists of four different types of events, according to quadrant analysis in the $u^{\prime}-w^{\prime}$ plane, they

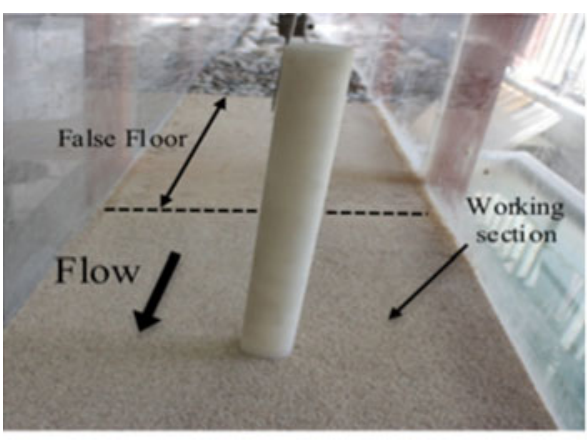

(a)

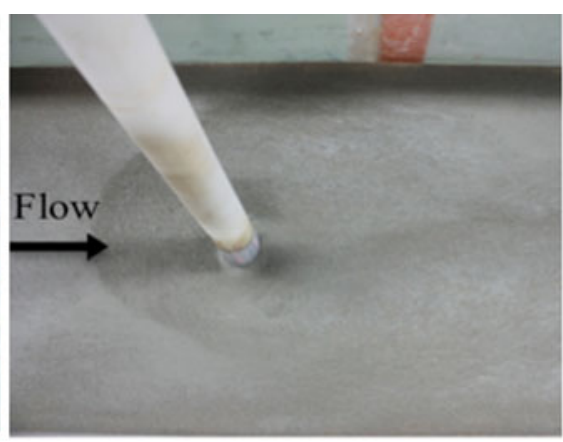

(b)

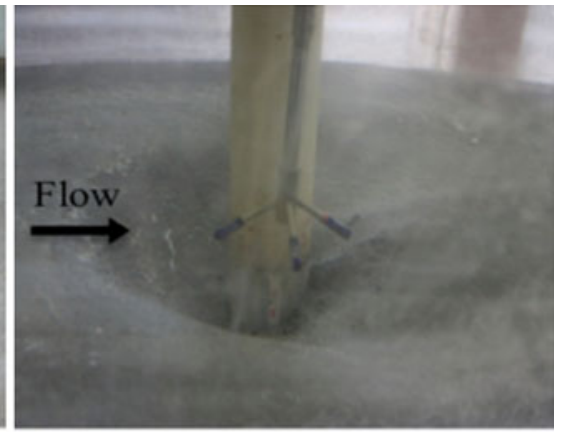

(c)

Fig. 1 (a) False floor glued with sediment and working section, (b) stabilizing the scour hole with cement, (c) measuring velocity profile with $\mathrm{ADV}$ 
are classified as: outward interaction $\left(u^{\prime}>0\right.$ and $\left.w^{\prime}>0\right)$, ejection $\left(\mathrm{u}^{\prime}<0\right.$ and $\left.w^{\prime}>0\right)$, inward interaction $\left(u^{\prime}<0\right.$ and $\left.w^{\prime}<0\right)$, and sweep $\left(u^{\prime}>0\right.$ and $\left.w^{\prime}<0\right) . u^{\prime}$ and $w^{\prime}$ are defined as follows,

$u^{\prime}=u-\bar{u}$

$w^{\prime}=w-\bar{w}$

where $u$ and $w$ are the instantaneous velocities in the longitudinal and vertical directions, respectively and $\bar{u}$ and $\bar{w}$ are the temporal mean velocities in the longitudinal and vertical directions. The temporal mean velocities are also given by,

$\bar{u}=\frac{1}{N} \sum_{i=1}^{N} u_{i}$

$\bar{w}=\frac{1}{N} \sum_{i=1}^{N} w_{i}$

where $N$ is the number of instantaneous velocity samples. The four quadrants identified for the velocity fluctuations and the associated bursting events are shown in Fig. 2.

The four events types have different effects on the rate, and mechanisms of sediment entrainment in a turbulent flow. An ejection event is characterized by an upward movement of low speed fluid, because the instantaneous local velocity is lower than the time-averaged local velocity. By the same definition, a sweep is a downward movement of high-speed fluid towards the bed. Therefore, bursting events in this quadrant have a significant influence on the entrainment of particles into the flowing water

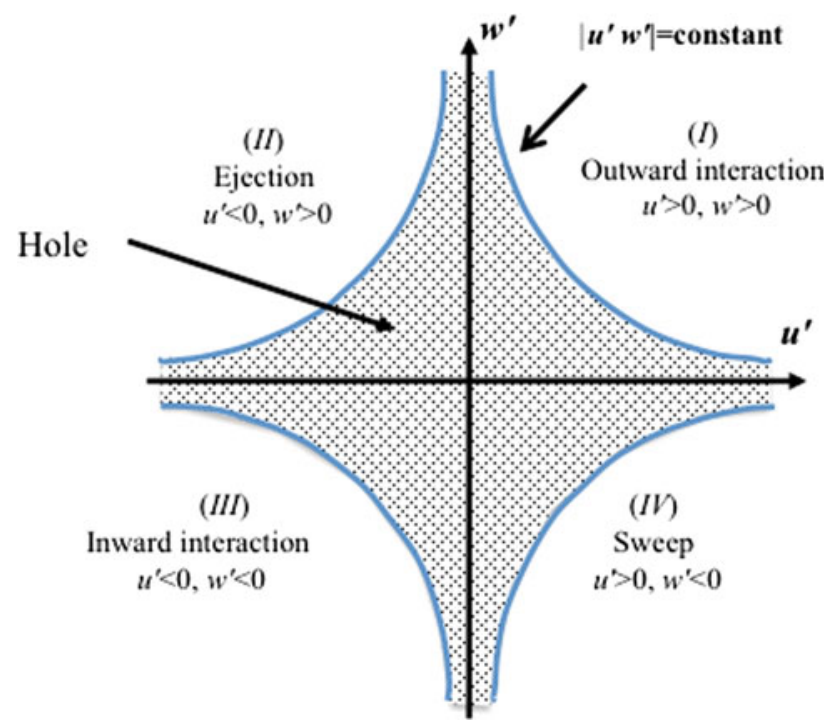

Fig. 2 Bursting events and their associated quadrants
(Keshavarzi and Ball 1997), while transport of suspended sediment occurs most frequently during ejection events.

A threshold level or hole size parameter $H$ is introduced in the $u^{\prime}-w^{\prime}$ plane for quadrant analysis. In Fig. 2, the cross-hatched region, called the "hole". It is bounded by the curves:

$\left|u^{\prime} w^{\prime}\right|=H \cdot \sqrt{\overline{u^{\prime 2}}} \cdot \sqrt{\overline{w^{\prime 2}}}$

where $H$ is the hole size. In quadrant analysis, events that are located in the hole are excluded. Zero $H$ indicates that all $u^{\prime}$ and $w^{\prime}$ are included in the analysis. By increasing parameter $H$, the progressively stronger events are eliminated. Therefore, a clear identification between strong and weak events is possible by the parameter $H$.

As pointed out by Nezu and Nakagawa (1993), the threshold level $H$, is more or less selected arbitrarily. In fact, the contribution of each quadrant to the Reynolds shear stress as a function of the threshold level of the hyperbolic hole region has been defined by (Dey and Nath 2010) as,

$\left\langle u^{\prime} w^{\prime}\right\rangle_{i, H}=\lim _{t \rightarrow \infty} \frac{1}{T} \int_{0}^{T} u^{\prime}(t) w^{\prime}(t) I_{H}^{i}(y, t) d t$

where, $T$ is time interval and $I_{H}^{i}(y, t)$ is a detection function defined as,

$I_{H}^{i}(y, t)= \begin{cases}1 & \text { when }\left|u^{\prime} w^{\prime}\right| \geq H\left|\overline{u^{\prime} w^{\prime}}\right| \\ 0 & \text { otherwise }\end{cases}$

The fractional contribution $S_{i, H}$ to $-\overline{u^{\prime} w^{\prime}}$ from each event is,

$S_{i, H}=\frac{\left\langle u^{\prime} w^{\prime}\right\rangle_{i, H}}{\left|\overline{u^{\prime} w^{\prime}}\right|}$.

For hole size (threshold level) $H=0$, the sum of contributions from all different bursting events is equal to one,

$\sum_{i=1}^{i=4} S_{i, 0}=1$.

The occurrence probability, for event type $i, P_{H}^{i}$ is computed as,

$$
\begin{aligned}
P_{H}^{i}= & \sum_{t=0}^{t=T_{M E A S}} I_{H}^{i}(y, t) / \sum_{t=0}^{t=T_{M E A S}}\left[I_{H=0}^{i=1}(y, t)+I_{H=0}^{i=2}(y, t)\right. \\
& \left.+I_{H=0}^{i=3}(y, t)+I_{H=0}^{i=4}(y, t)\right]
\end{aligned}
$$

where $T_{M E A S}$ is measurement-time length. Note that the occurrence probability is normalized by the total number of data by using $H=0$ as index in the denominator of Eq. (9) (Cellino and Lemmin 2004). 


\section{Results and discussions}

\subsection{Velocity profiles}

For the analysis of the velocity profiles and turbulence quantities, the instantaneous three dimensional velocity components were measured by ADV around the pier. Due to the shape of ADV (three receiving transducers mounted on short arms around the transmitting transducer at $120^{\circ}$ azimuth intervals) the closest distance for measuring the velocity was $2 \mathrm{~cm}$ from the pier boundary.

The velocity measurements, around the circular pier in the scour hole, have confirmed the complex three-dimensional pattern of flow around the pier. The flow at upstream of the pier is characterized by a flow circulation and a strong downward flow at regions close to the pier. In downstream of the pier, the flow circulation remains however with a diminishing strength. In downstream of the pier, there is a flow reversal towards the pier close to the surface. As the flow moves downstream, by leaving the scour hole, the flow reversal diminishes and the flow is recovering to approach the flow condition. The measurements around the pier show that the flow in the far region beyond the scour hole circumference does not change with the presence of the pier. Dey and Rajkumar (2007b) investigated the characteristics of horseshoe vortex at upstream of the pier at different stages during the progress of the scouring process. For a circular pier, they pointed out that the maximum down-flow occurs very close to the upstream of the pier in equilibrium scour hole. Istiarto (2001) concluded that the flow is altered only in the scour hole region.

Upstream and downstream of the pier are the two critical points according to previous studies (Dey and Rajkumar 2007b; Istiarto 2001), thus these two points were considered as instances for detail investigation in the following sections. The profile of the dominant component of velocity $u$ is shown in Fig. 3 at upstream and downstream of the pier. As indicated in Fig. 3a, e for upstream of the pier, reversed velocity occurs near the base. Thus, it verifies that a strong horseshoe vortex exists upstream of the pier inside the scour hole. This is in agreement with the result of Dey and Rajkumar (2007b). They pointed out the strongest vertical flow is observed at upstream of the pier.

The time-averaged absolute velocity $V=\left(u^{2}+v^{2}+w^{2}\right)^{0.5}$ at upstream and downstream of the pier is depicted in Fig. 3e. The vector plot displays the complicated turbulence flow at upstream and downstream of the pier. At upstream of the pier and above the scour hole, the absolute velocity $V$ is uniform and the vortical flow is not observed. By decreasing the distance from the bed, the intensity of the down flow increases. The reversal flow is clearly visible at upstream of the pier (in the scour hole) and at downstream of the pier (above the scour hole).

From Fig. 3a, e it can be observed that by increasing the distance from the bed the stream-wise component of velocity becomes positive in the scour hole. The maximum velocity occurs roughly on the edge of scour hole and the velocity profile becomes uniform out of the scour hole.

For the ratio of the flume width to flow depth (aspect ratio) less than 5 , dips in velocity profiles have been observed over smooth beds by Nezu et al. (1989) and over rough beds by Kironoto and Graf (1994). Graf and Altinakar (1998) inferred that secondary currents cause the "dip phenomenon" of velocity profiles. They indicated that for an aspect ratio of $W / h>5$, the maximum flow velocity occurs at the water surface and the effect of the secondary currents could be eliminated; whereas for an aspect ratio of $W / h<5$, the influence of the secondary currents is significant and the maximum velocity occurs under the water surface. In the present study, an aspect ratio is less than $5(W / h=2.5)$. In addition the existence of the pier in the flow intensifies the secondary currents and displaces the location of the maximum velocity toward the bed.

At downstream of the pier, the velocity increases and approaches to its maximum value at $z / h$ approximately equal to 0.1 inside the scour hole (see Fig. 3b, e). In this region, the velocity reversal phenomenon occurs out of the scour hole toward the water surface. By comparing the maximum velocity at upstream and downstream of the pier, it can be concluded that secondary currents are more significant at downstream of the pier because the maximum velocity occurs near the bed in this region.

In uniform flows, the universal log-law can describe velocity profile for the inner layer $(z / h \leq 0.2)$ (Graf and Altinakar 1998),

$\frac{u(z)}{u_{*}}=\frac{1}{\mathrm{~K}} \ln \left(\frac{z}{k_{s}}\right)+B_{R}$

where $u_{*}$ is the friction velocity, $k_{s}$ is the equivalent standard roughness of the bed $\left(k_{s}=d_{50}=0.72 \mathrm{~mm}\right), B_{R}$ is a constant of integration, and $\kappa$ is the Von Karman constant $(\kappa=0.4)$. At upstream of the pier the reversal flow occurs near the bed. By approaching to the water surface the stream-wise component of velocity becomes positive in the scour hole. The universal log-law is valid for this part (see Fig. 3c). Consequently, after eliminating the negative velocities in inner layer that occur in the separation region near the bed, the velocity profile in the scour hole becomes logarithmic. As shown in Fig. 3d the logarithmic distribution is not valid at downstream of the pier due to the reversal flow near the water surface. 

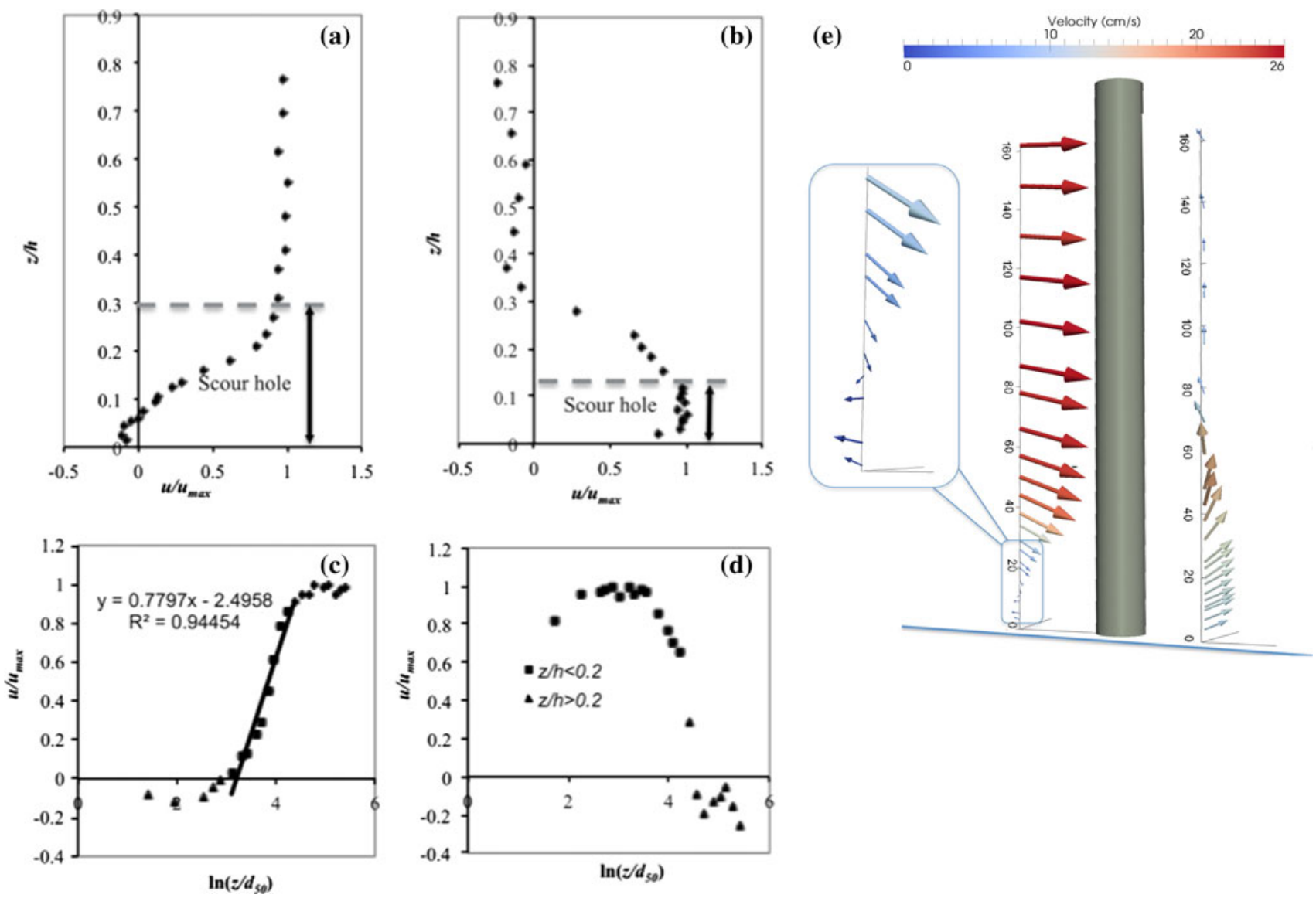

Fig. 3 Velocity profiles; (a) upstream of the pier, (b) downstream of the pier, (c) universal law of the wall for stream-wise velocity fit for upstream of the pier, (d) universal law of the wall for stream-wise

In order to estimate the correlation between the velocities at upstream and downstream of the pier the correlation coefficient, $R$, is calculated which is defined as:

$R=\frac{\sum(X-\bar{X})(Y-\bar{Y})}{\sqrt{(X-\bar{X})^{2}} \sqrt{(Y-\bar{Y})^{2}}}$

in which $X$ and $Y$ are measured velocities at the same distance from the bed at upstream and downstream of the pier, respectively. $\bar{X}$ and $\bar{Y}$ are the corresponding mean velocities in upstream and downstream of the pier.

The correlation coefficient between velocity distribution in upstream and downstream of the pier was obtained to be $R=-0.88$. Since the absolute value of correlation coefficient is more that $0.7(|R|>0.7)$, the strong correlation exists between velocities in upstream and downstream of the pier. Negative correlation defines a relationship between two variables in which one variable increases as the other decreases, and vice versa. In statistics, a perfect negative correlation is represented by the value $R=-1$, while $R=0$ indicates no correlation. Therefore it can be concluded that these two variables may be negatively correlated in some, but not all, cases. As is also clear in Fig. 3.

velocity for downstream of the pier, (e) time averaged absolute velocity. Magnitudes of velocities are shown via a color code (Color figure online)

\subsection{Turbulence intensities}

The turbulence intensities are the Root-Mean-Square (RMS) values of the velocity fluctuations. Figure 4 shows the vertical profiles of the turbulence intensities upstream and downstream of the pier. At upstream of the pier and close to the bed, RMS $\left(u^{\prime}\right)$ has decreasing tendency due to the reversal flow near the bed. All the three components of turbulence intensities increase up to the edge of scour hole (maximum velocity) and then decrease and finally approach a roughly constant value.

As indicated in Fig. 4, in the scour hole and at upstream of the pier, $\operatorname{RMS}\left(v^{\prime}\right)$ is larger than $\operatorname{RMS}\left(u^{\prime}\right)$ and $\operatorname{RMS}\left(w^{\prime}\right)$. This indicates that the secondary currents are dominant in this region. Above the scour hole RMS of the stream-wise velocity $\left(u^{\prime}\right)$ values are larger than RMS of span-wise $\left(v^{\prime}\right)$ and vertical $\left(w^{\prime}\right)$ velocities due to decrease of the secondary currents and uniformity of the velocity profile.

As a result of flow separation, the core of the high turbulence intensity is in downstream of the pier. The turbulence intensity decreases by increasing the distance from the bed. The maximum stream-wise and span-wise and vertical turbulence intensities occur at the edge of the score hole. According to the Fig. 4 
Fig. 4 Profiles of turbulence intensities at upstream and downstream of the pier

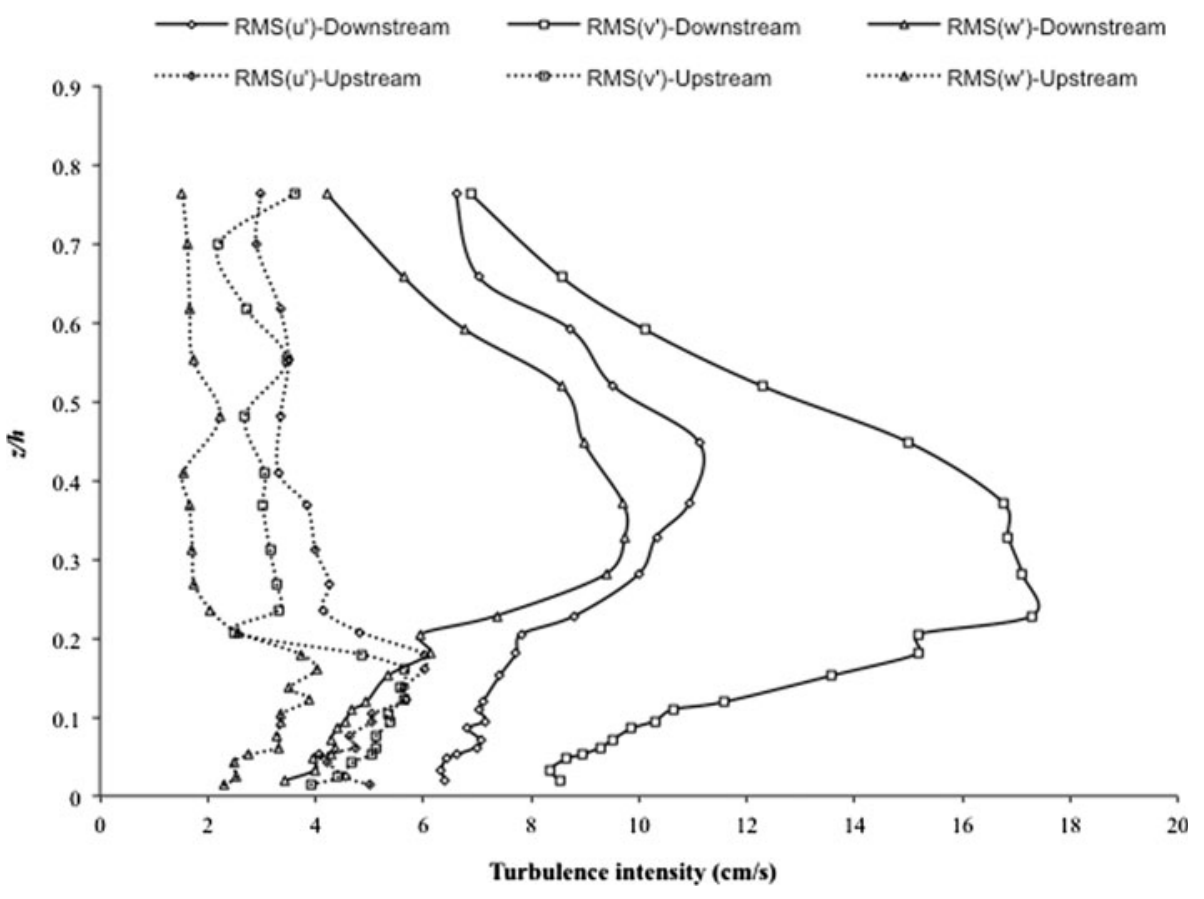

at downstream of the pier, and over the entire flow depth, the span-wise turbulence intensity is stronger than the stream-wise and vertical directions due to the flow separation at downstream of the pier and also because of dominance of the wake vortex in this region. Since $\operatorname{RMS}\left(v^{\prime}\right)$ is stronger at downstream of the pier, the secondary currents are more dominant in this region. Consequently, in downstream of the pier the maximum velocity occurs at a location, which is closer to the bed.

\subsection{Reynolds shear stress}

The profiles of Reynolds shear stresses $-\overline{u^{\prime} w^{\prime}}$ and $-\overline{v^{\prime} w^{\prime}}$ are shown in Fig. 5. In general, the vertical Reynolds stress $-\overline{u^{\prime} w^{\prime}}$ at downstream of the pier is stronger than upstream of the pier. Fluctuations observed in Fig. 5 are mainly due the flow separation and vortex shedding.

Because of the reversal flow and also the dominance of the secondary currents, the distribution of $-\overline{v^{\prime} w^{\prime}}$ is negative at downstream of the pier. In this region the magnitude of $-\overline{v^{\prime} w^{\prime}}$ is larger as compared to upstream of the pier. From Fig. 5 it can be observed that at upstream of the pier, near the scoured bed, the $-\overline{u^{\prime} w^{\prime}}$ becomes negative due to the return flow. In this region, values of $-\overline{u^{\prime} w^{\prime}}$ and $-\overline{v^{\prime} w^{\prime}}$ above the scour hole are almost constant.

The standard deviations of instantaneous Reynolds shear stress are plotted in Fig. 6a at upstream (u) and downstream (d) of the pier. As it can be observed Reynolds shear stresses are fluctuating heavily at downstream of the pier as a result of wake vortices in this region. To get further insight about the probability distribution of instantaneous Reynolds shear stresses we measure the non-Gaussian parameter, $\alpha$, defined from the ratio of the forth moment to the second moment of the distribution as (Vorselaars et al. 2007),

$\alpha=\frac{<X^{4}>}{3<X^{2}>^{2}}-1$

where $X$ is the value of the shear stress and $<>$ denotes averaging over all members of the ensemble. For a Gaussian distribution $\alpha$ is zero. The non-Gaussian parameter for the Reynolds shear stresses are however larger than zero at upstream and down stream of the pier, meaning that they are characterized by a distribution whose tails decay to zero slower than a Gaussian distribution. Deviation from the Gaussian distribution can also be observed in quantilequantile plot (QQ-plot) of the quantiles of the Reynolds shear stress values versus theoretical quantiles from a Gaussian distribution (Chambers et al. 1983). If the distribution of Reynolds shear stress is Gaussian, the QQ-plot will be close to a linear form. Figure $6 \mathrm{~b}$ displays the QQ-plot of the $-\overline{u^{\prime} w^{\prime}}$ at upstream of the pier for $z / h=0.2$ as an example. The shape of the curve deviates from the dashed line and therefore it confirms that the tails of the shear stress distributions are not Gaussian.

\subsection{Frequency of event}

In order to apply bursting analysis for determining the contribution of each quadrant to the Reynolds shear stress, 
Fig. 5 Profiles of Reynolds stress at upstream and downstream of the pier
Fig. 6 (a) Standard deviations of instantaneous Reynolds shear stress at upstream (u) and downstream (d) of the pier; (b) QQ-plot of the at upstream of the pier for $z / h=0.2$
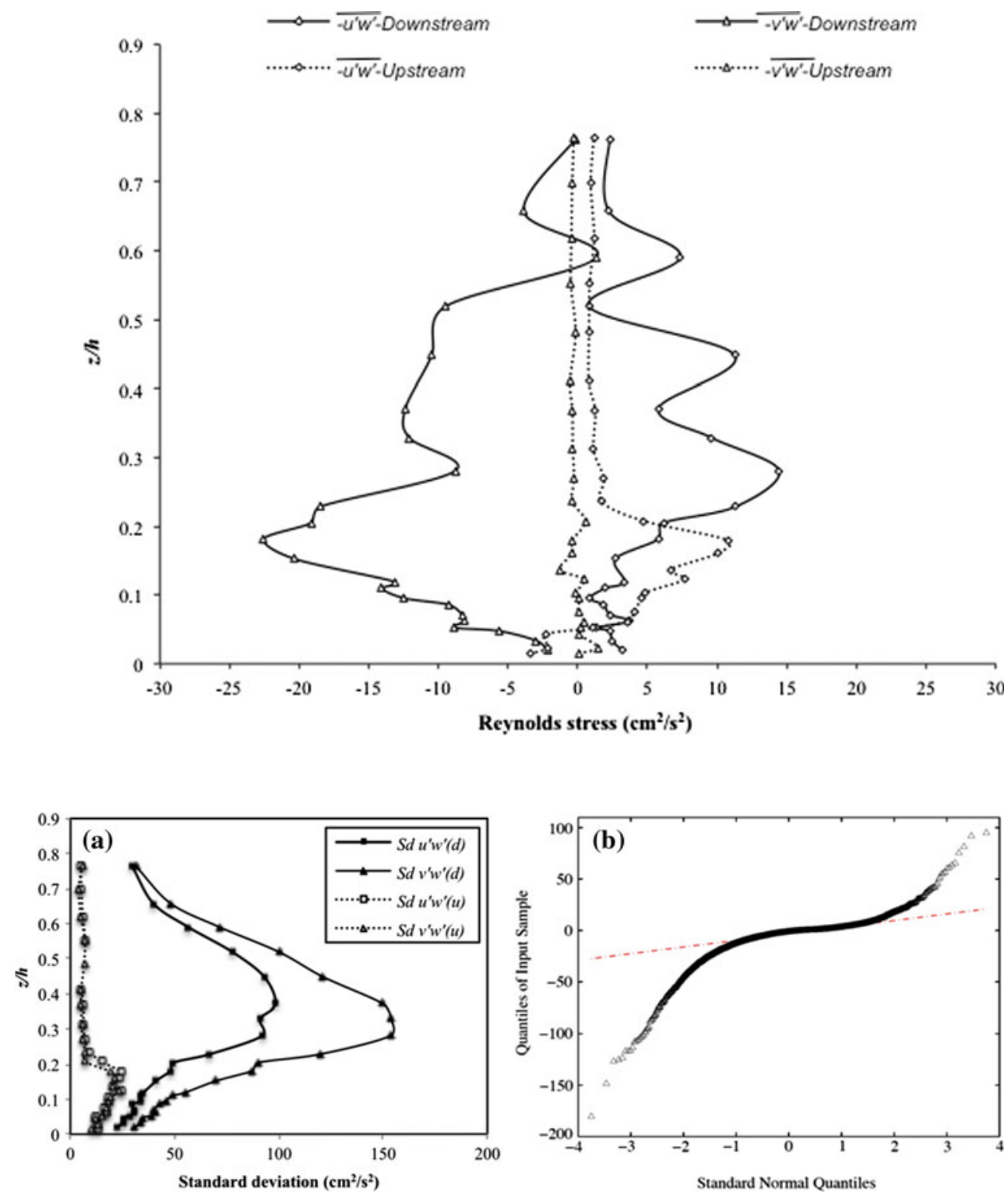

a computer program was written in $M A T L A B$. The fractional contribution of $S_{i, H}$ to the Reynolds shear stress for each quadrant was determined by varying the hole size parameter $H$.

Several values of the hole size $H$ have been considered in the literature. The hole size $H$ was chosen in the range of 4-4.5 by Lu and Willmarth (1973) for ejection events. Beyond this level, the contributions of sweep events to the Reynolds stress become negligible. Mazumder et al. (2009) have chosen $H=0,2,5,6,10,15$ in quadrant analysis over bedforms. Afzalimehr et al. (2011) considered $H=0$, $1,2,3$ to investigate the characteristics of turbulent flow in channel with dense vegetation. A wide range of hole sizes, $H=0,1,2 \ldots 10,15,20$ are considered in order to recognize the differences between the weak and strong events in bursting process. The computation has been performed by first taking into account all the events $(H=0)$, and then progressively stronger fractional events have been selected.

The occurrence probability profiles are shown in Fig. 7 at upstream and downstream of the pier for $H=0$. It is important to note that equilibrium scour depth in upstream of the pier is located at $z / h=0.3$. As shown in Fig. 7a, inside the scour hole for $z / h<0.05$ the highest occurrence probabilities are found for outward interactions and inward interactions rather than ejection and sweep. Thus, in the scour hole near the bed, flow does not have enough energy to transport particles; hence sedimentation occurs in this region. As can be seen also visually during the experiment, the particles in scour hole near the bed are lifted up. However, they can't move along the flow and finally they return to the bed.

For $z / h>0.05$, the dominant events are ejection and sweep. Outside the scour hole $(z / h>0.3)$, in outer region, 
the fluctuations of all probabilities are more modest compared to their fluctuations inside the scour hole. The depthaveraged values of the occurrence probabilities in upstream of the pier indicates that sweep has highest occurrence probabilities. It is followed by ejection, outward interaction and inward interaction events. Sweep event is the most important bursting event for entrainment of sediment particles and causes movement of particles by rolling, sliding, and saltation; consequently, the maximum scour depth takes place at upstream side of the pier and sediment transports in downstream direction.

In downstream of the pier, near the bed, the occurrence probabilities of ejection are more than sweep events. Thus, the sediment become suspended in this region. The occurrence probabilities of ejection decrease toward water surface. The occurrence probabilities of ejection and sweep events become nearly the same in outer region. The other two events, outward interactions and inward interactions, have nearly the same occurrence probabilities. Due to their intensity, ejections and sweeps contribute strongly to instantaneous shear stress. For this reason, these events are expected to play an important role in the erosion, transport, and deposition of particles in sediment transport (Cellino and Lemmin 2004).

To assess the influence of $H$ on $S_{i, H}$, contributions of $S_{i, H}$ to the Reynolds shear stress in each quadrants are plotted in the entire flow depth at upstream of the pier for $H=0,10$,
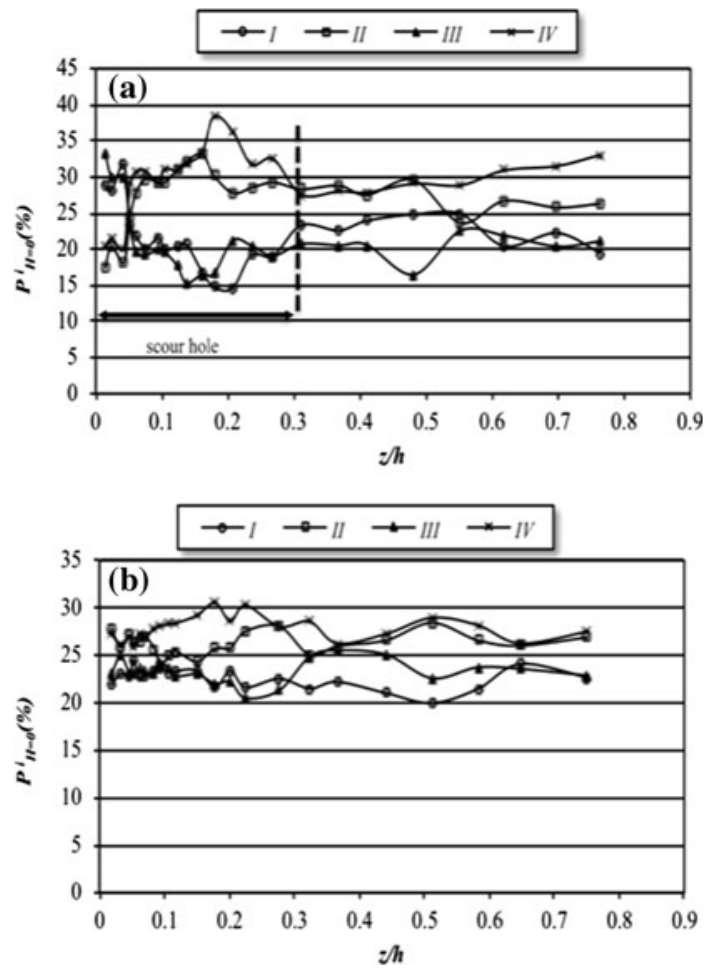

Fig. 7 Occurrence probability of event types for each quadrant $(I, I I, I I I, I V)$; (a) upstream of the pier, (b) downstream of the pier
20 in Fig. 8a-c. For $H=0$ (see Fig. 8a), since no value of Reynolds stress is eliminated in four quadrants, trend of Fig. 8a is similar to Fig. 7a. Near the bed, outward interaction and inward interaction are dominant. By increasing the distance from the bed, the trend changes and ejection and sweep become dominant.

By increasing the hole size $H$, the contribution of each event to the shear stress becomes comparable and the fluctuation of $S_{i, H}$ over the flow depth is suppressed. As shown in Fig. 8, in the region with $z / h \leq 0.2$ by increasing $H$ the fluctuations of $S_{i, H}$ over the flow depth are suppressed faster compared to the region with $z / h \leq 0.2$. Therefore, the weak events are much more dominated at $z / h \leq 0.2$

In Fig. 9 the contribution of each quadrant $\left(S_{i, H}\right)$ to the Reynolds shear stress is illustrated for the entire flow depth at downstream of the pier for $H=0,10,20$. At downstream of the pier, regardless the hole size $(H)$, both ejection $\left(S_{2, H}\right)$ and sweep $\left(S_{4, H}\right)$ contribute significantly to the Reynolds shear stress. Although with increasing hole size the discrimination between each events become more difficult, but the dominant event is ejection $\left(S_{2,20}\right)$ that is followed by sweep $\left(S_{4,20}\right)$. At downstream of the pier, the fluctuations of $S_{i, H}$ over the flow depth is stronger as compared to the upstream side of the pier. It can be concluded that by increasing the hole size, $H$, the weak events aren't completely excluded at downstream of the pier, in contrast to the upstream side of the pier.

The variation of the fractional contributions, $\left|S_{i, H}\right|$, as a function of the hole size $H$ for each of the four quadrants at $z / h=0.2$ (near the edge of scour hole) at upstream and downstream of the pier are shown in Fig. 10. At $z / h=0.2$, quadrant (II) and (IV) events are dominant and quadrant (I) and (III) events appear to contribute weakly to the Reynolds shear stress production. Generally by increasing the hole size, the contribution of each event to the Reynolds shear stress generation becomes small.

As indicated in Fig. 10, upstream of the pier at $z / h=$ 0.2 , ejection $\left(\left|S_{2,0}\right| \approx 0.8\right)$ is the dominant event which is followed by sweep $\left(\left|S_{4,0}\right| \approx 0.6\right)$. The contribution of sweep on the Reynolds shear stress production becomes negligible for $H>10$ whereas the contribution of ejection is significant even at $H>20$. On the other hand, the contributions of outward and inward interaction to the Reynolds shear stress are rather weak $\left(\left|S_{1,0}\right|=\left|S_{3,0}\right| \approx\right.$ $0.2)$. These contributions vanish when $H>6$ and $H>10$ for quadrant (III) and (I) respectively.

At downstream of the pier, the contribution of each event to the Reynolds shear stress production is more significant than upstream of the pier. In this region, at $z / h=0.2$, the contributions of ejection and sweep become comparable $\left(\left|S_{2,0}\right|=\left|S_{4,0}\right| \approx 1.4\right)$ and both are greater 
Fig. $8 S_{i, H}$ versus $z / h$ for different values of $H$ upstream of the pier for each quadrant $(I, I I, I I I, I V)$

Fig. $9 S_{i, H}$ versus $z / h$ for different values of $H$ downstream of the pier for each quadrant $(I, I I, I I I, I V)$
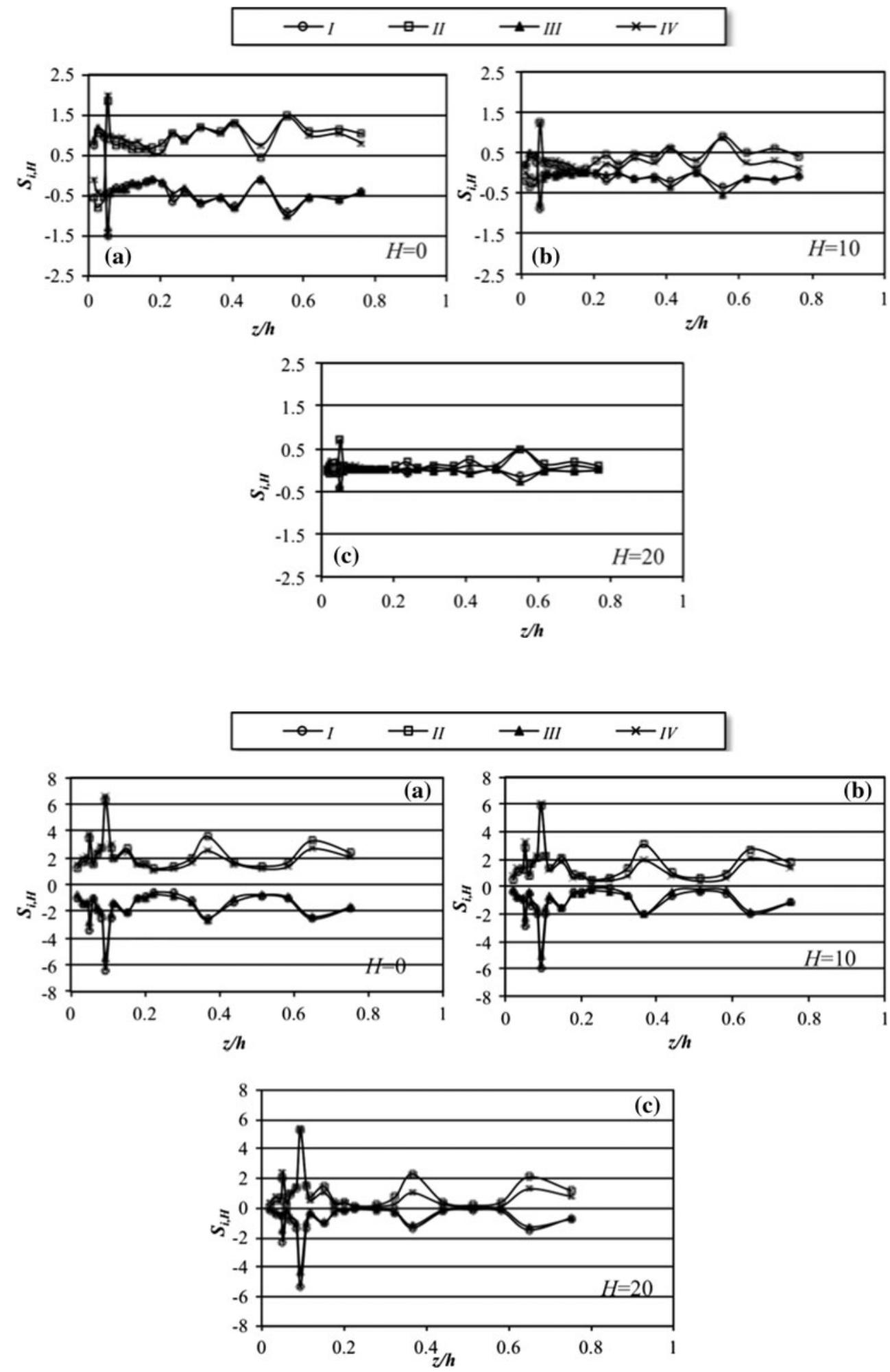

than outward interaction $\left(\left|S_{1,0}\right| \approx 0.9\right)$ and inward interaction $\left(\left|S_{3,0}\right| \approx 1\right)$. For each of the four quadrants at $z / h=$ 0.2 in downstream of the pier the contribution of each events to the Reynolds shear stress is still significant for hole sizes as large as $H>20$. Therefore, at downstream of the pier, although quadrant $(I I)$ and $(I V)$ are more significant, each of the four quadrants has important effects on Reynolds shear stress. 
Fig. 10 Variation of $\left|S_{i, H}\right|$ with $H$ for $z / h=0.2$ at upstream and downstream of the pier for each quadrant $(I, I I, I I I, I V)$

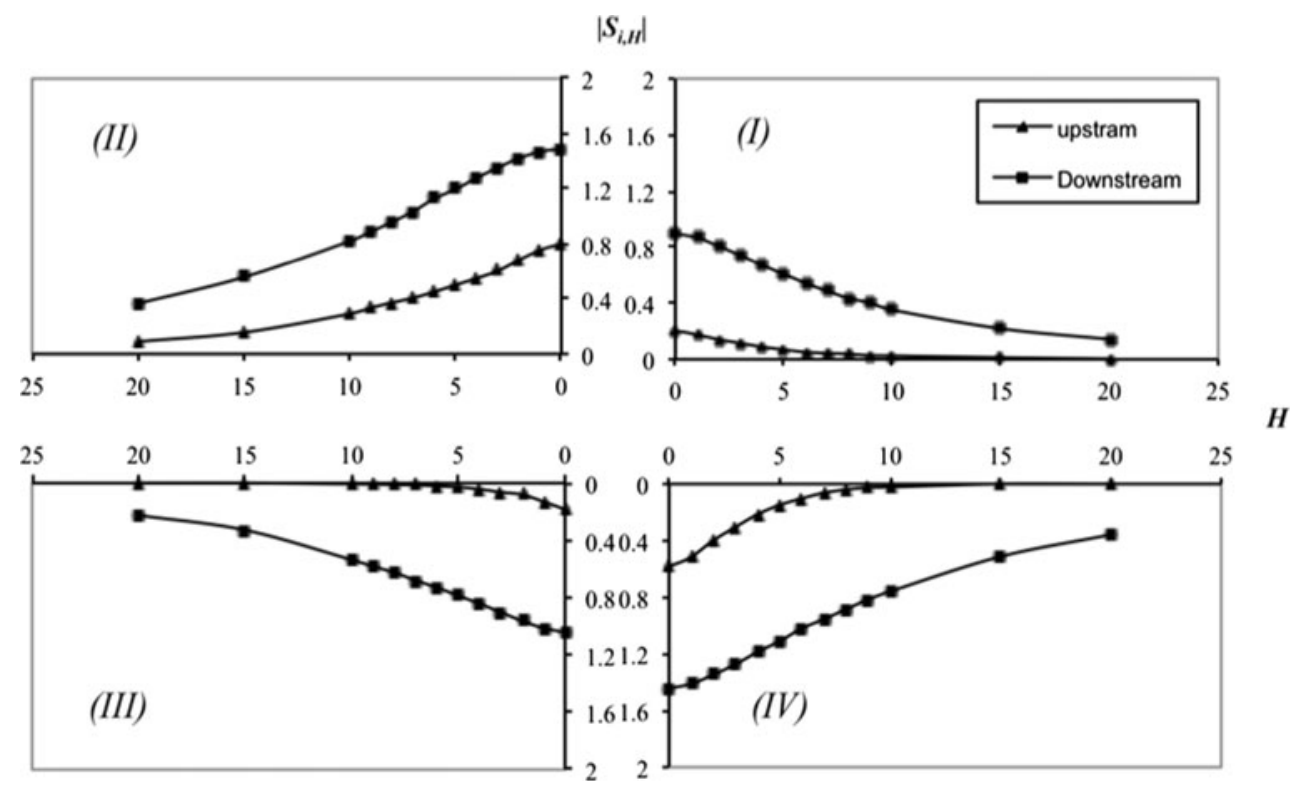

\section{Conclusions}

The structure of turbulent flow over the scour hole is important for understanding the sediment particle entrainment and transport. Two important issues that need to be understood in sediment movements are the stochastic nature of instantaneous shear stresses over the scour hole and its influences on sediment entrainment and transport. In the present study, stochastic nature of turbulent flow around a bridge pier under a clear water regime has been investigated experimentally. The results indicate that probability distribution of instantaneous Reynolds shear stresses are characterized by a distribution whose tails decay to zero slower than a Gaussian distribution. Reynolds shear stresses are fluctuating heavily at downstream of the pier as a result of wake vortices in this region. Unsteady shedding wake vortices are created due to the separation of the flow at downstream of the pier. These wake vortices are very unsteady and oriented approximately vertically with low pressure at the vortex cores. These vortices act like small tornadoes, lifting up sediment particles and transporting the suspended sediment toward downstream. The measurements reveal that at upstream of the pier the maximum velocity occurs roughly on the edge of scour hole. Outside the scour hole, the velocity profile becomes uniform. The vertical profile of its longitudinal velocity component at upstream of the pier, after eliminating the negative velocities occurring in the separation region near the bed, can be well explained by the logarithmic law of the wall for the inner region. It was found that the secondary currents are more significant at downstream of the pier because the maximum velocity occurs closer to the bed. Similarly RMS $\left(v^{\prime}\right)$ is larger in this region compared to the upstream of the pier. In general, at downstream of the pier, the vertical Reynolds stress $\left(-\overline{u^{\prime} w^{\prime}}\right)$ is stronger than the upstream side of the pier. The quadrant analysis indicate that at upstream of the pier, near the bed, outward interaction and inward interaction are dominant. By increasing the distance from the bed, the trend changes and ejection and sweep become dominant. This change is due to the velocity reversal phenomenon that occurs near the bed at upstream of the pier. Inside the scour hole near the bed, flow does not have enough energy to transport particles; hence sedimentation occurs in this region. Particles in the scour hole near the bed are lifted up, however they can't move along the flow and finally they return to the bed. By comparing depth averaged values of the occurrence probabilities at upstream of the pier, it was found that the occurrence probability of sweep events is more than other events, which followed, by ejection, outward interaction and inward interaction. Since the sweep events are dominant phenomena at upstream of the pier, the maximum scour depth takes place at this region and eroded sediment are transported in downstream direction. An assessment of the variation of $\left|S_{i, H}\right|$ with $H$ at upstream and downstream of the pier reveals that the contribution of each event to the Reynolds shear stress production at downstream of the pier is stronger.

\section{References}

Afzalimehr H, Moghbel R, Gallichand J, Sui J (2011) Investigation of turbulence characteristics in channel with dense vegetation. Int $\mathrm{J}$ Sed Res (IJSR) 26(3):269-282

Arlinghaus R, Engelhardt C, Sukhodolov A, Wolter C (2002) Fish recruitment in a canal with intensive navigation, Implications for ecosystem management. J Fish Biol 61:1386-1402 
Armstrong JD, Kemp PS, Kennedy GJA, Ladle M, Milner NJ (2003) Habitat requirements of Atlantic salmon and brown trout in rivers and streams. Fish Res 62:143-170

Barbhuiya AK, Dey S (2004) Local scour at abutments: a review. Acad Sci 29:449-476

Cellino M, Lemmin U (2004) Influence of coherent flow structures on the dynamics of suspended sediment transport in open channel flow. J Hydraul Eng (ASCE) 130(11):1077-1088

Chambers JM, Cleveland WS, Kliener B, Tukey PA (1983) Graphical methods for data analysis. Duxbury Press, Boston

Chiew YM (1992) Scour protection at bridge piers. J Hydraul Eng (ASCE) 118(11):1260-1269

Chiew YM, Lim FH (2000) Failure behavior of riprap layer at bridge piers under live-bed conditions. J Hydraul Eng (ASCE) 126(1): 43-55

Chiew YM, Melville BW (1987) Local scour around bridge piers. J Hydraul Res 25(1):15-26

Dey S (1997) Local scour at piers, part 1: A review of development of research. Int J Sed Res (IJSR) 12(2):23-44

Dey S, Nath T (2010) Turbulence characteristics in flows subjected to boundary injection and suction. J Eng Mech (ASCE) 136(7): 877-887

Dey S, Rajkumar VR (2007a). Clear-water scour at piers in sand beds with an armor layer of gravels. J Hydraul Eng (ASCE) 133(6): 703-711

Dey S, Rajkumar VR (2007b). Characteristics of horseshoe vortex in developing scour holes at piers. J Hydraul Eng (ASCE) 133(4):399-413

Dey S, Bose SK, Sastry GLN (1995) Clear water scour at circular piers: a model. J Hydraul Eng (ASCE) 121(12):869-876

Esfahani FS, Keshavarzi A (2011) Effect of different meander curvatures on spatial variation of coherent turbulent flow structure inside ingoing multi-bend river meanders. J Stoch Environ Res Risk Assess 25:913-928

Ghisalberti M, Nepf H (2006) The structure of the shear layer over rigid and flexible canopies. Environ Fluid Mech 6(3):277-301

Graf W H, Altinakar MS (1998) Fluvial hydraulics: Flow and transport processes in channels of simple geometry. Willey, Chichester, ISBN 0-471-97714-4

Grass AJ (1971) Structural features of' turbulent flow over smooth and rough boundaries. J Fluid Mech 50(2):233-255

Grass AJ (1982) The influence of boundary layer turbulence on the mechanics of sediment transport. In: Proceedings of Euromech 156. Mechanics of sediment transport, Istanbul, pp 3-17

Istiarto, I. (2001). Flow around a cylinder in a scoured channel bed. PhD-thesis, Nr 2368, Ecole Polytechnique Fédérale de Lausanne, Switzerland

Jafari Mianaei S, Keshavarzi A (2010) Study of near bed stochastic turbulence and sediment entrainment over the ripples at the bed of open channel using image processing technique. J Stoch Environ Res Risk Assess 24:591-598

Keshavarzi AR, Ball JE (1997) An analysis of the characteristics of rough bed turbulent shear stress in an open channel flow. J Stoch Hydrol Hydraul 11:193-210

Keshavarzy A, Ball JE (1995) Instantaneous shear stress on the bed in a turbulent open channel flow. Proceedings of XXVI IAHR congress, London

Kironoto BA, Graf WH (1994) Turbulence characteristics in rough uniform open channel flow. Proc ICE Water Maritime Energy 106:333-344
Kline SJ, Reynolds WC, Sehraub FA, Runstadler PW (1967) The structure of turbulent boundary layers. J Fluid Mech 30(4): $741-773$

Kummar V, Ranga Raju KG, Vittal N (1999) Reduction of local scour around bridge piers using slot and collar. J Hydraul Eng (ASCE) 125(12):1302-1305

Lu SS, Willmarth WW (1973) Measurements of the structures of the Reynolds stress in a turbulent boundary layer. J Fluid Mech 60(3):481-511

Mazumder BS, Pal DK, Ghoshal K, Ojha SP (2009) Turbulence statistics of flow over isolated scalene and isosceles triangularshaped bedforms. J Hydraul Res 47(5):626-637

Melville BW (1975) Local scour at bridge sites. Rep. No. 117, School of Engineering, University of Auckland, Auckland, New Zealand

Melville BW, Chiew YM (1999) Time scale for local scour at bridge piers. J Hydraul Eng (ASCE) 125(1):59-65

Melville BW, Coleman SE (2000) Bridge scour. Water Resources Publications LLC, Littleton Colo

Mia Md F, Nago H (2003). Design method of time-dependent local scour at a circular bridge pier. J Hydraul Eng 129(6):420-427

Moncada-M AT, Aguirre PEJ, Bolívar JC, Flores EJ (2009) Scour protection of circular bridge piers with collars and slots. J Hydraul Res 47(1):119-126

Nakagawa H, Nezu I (1978) Bursting phenomenon near the wall in open channel flows and its simple mathematical model. Mem Fac Eng Kyoto Univ XL(4) 40:213-240

Nezu I, Nakagawa H (1993) Turbulence in open channel flows. IAHR Monograph, Balkema, Rotterdam

Nezu I, Nakagawa H, Rodi W (1989) Significant difference between secondary currents in closed channels and narrow open channel. In: The 23rd IAHR congress, Ottawa, Canada, pp 125-132

Poggi D, Porporato A, Ridolfi L, Albertson JD, Katul GG (2004) The effect of vegetation density on canopy sub-layer turbulence. Boundary-layer Meteorol 111:565-587

Raudkivi AJ (1990) Loose boundary hydraulics, 3rd edn, Pergamon, Oxford

Raudkivi A, Ettema R (1983) Clear water scour at cylindrical piers. J Hydraul Eng 109(3):338-350

Thorne PD, Williams JJ, Heathershaw AD (1989) In-situ acoustic measurements of marine gravel threshold and transport. Sedimentology 36:61-74

Vorselaars B, Lyulin AV, Karatasos K, Michels MAJ (2007) NonGaussian nature of glassy dynamics by cage to cage motion. Phys Rev E 75:011504

Wallace JM, Eckelmann H, Brodkey RS (1972) Structure of the Reynolds stress near the wall. J Fluid Mech 54:65-92

Williams JJ (1990) Video observations of marine gravel transport. Geo Mar Lett 10:157-164

Willmarth WW, Lu SS (1972) Structure of Reynolds stress near the wall. J Fluid Mech 55:65-92

Zarrati AR, Gholami H, Mashahir MB (2004) Application of collar to control scouring around rectangular bridge piers. J Hydraul Res 42(1):97-103

Zhu W, van Hout R, Katz J (2007) PIV measurements in the atmospheric boundary layer within and above a mature corn canopy. Part B: Quadrant-hole analysis. J Atmos Sci 64: $2825-2838$ 